\title{
Expertises en médecine des assurances
}

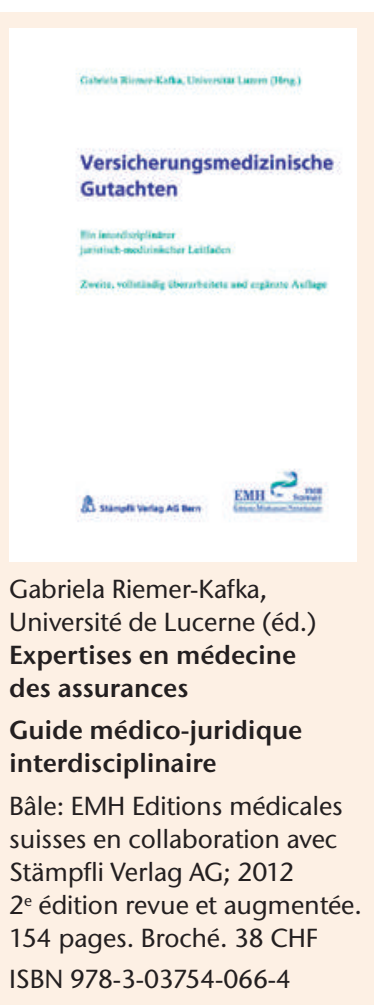

L'expertise en médecine des assurances est un moyen de preuve utilisé dans les affaires litigieuses par les assurances, les tribunaux ou les parties concernées. Étant donné que la prise d'une décision correcte dépend de la plausibilité et de la consistance de l'expertise, il convient de définir les exigences matérielles et formelles relatives à cette dernière.

Le présent guide juridico-médical se distingue par le fait que des médecins et des juristes ont élaboré en commun les questions soulevées par l'établissement de rapports d'expertise. Il traite de la fonction et des modalités de l'expertise, des tâches de l'expert et des concepts spécifiques à l'expertise.

L'éditrice, professeure en droit des assurances sociales et du travail à l'Université de Lucerne et initiatrice d'un cercle d'études interdisciplinaire de médecins et de juristes, est autrice de nombreuses publications en droit des assurances sociales. Elle est ainsi qualifiée pour publier un tel ouvrage interdisciplinaire. L'expérience nous ayant montré que juristes et médecins pensent et argumentent en principe de façon différente, la tentative d'une synthèse de ces deux pensées doit être qualifiée de positive.

L'objectif de ce groupe interdisciplinaire consistait en l'analyse de questions ouvertes et de difficultés de compréhension entre juristes et médecins et en l'harmonisation des attentes réciproques concernant la qualité des expertises médicales. Le présent guide remanié constitue le résultat de cette collaboration.

Le premier chapitre présente la fonction de l'expertise médicale et les tâches de l'expert. Dans un pre- mier temps, la relation médecin-patient, la méthodologie de l'expertise et les exigences relatives aux experts et aux expertises sont exposées du point de vue médical, puis les rapports juridiques entre patient, décideur et expert sont examinés, suivis de la jurisprudence du Tribunal fédéral avec les lignes de conduite consécutives concernant la valeur probante des expertises médicales et leur appréciation en tant que preuves.

Le deuxième chapitre discute de façon approfondie les modalités spécifiques d'une expertise en médecine des assurances. Il aborde également des questions relatives aux aspects temporels de l'expertise, à la procédure optimale pour les patients de langue étrangère et à la protection des données.

Dans le dernier chapitre, une sélection de concepts importants est récapitulée dans une perspective médicale et juridique.

Dans l'ensemble, le guide donne un bon aperçu, quasi complet, de l'expertise en médecine des assurances, favorise la compréhension de la pensée juridique pour les médecins et vice versa, et sert avant tout d'ouvrage de référence pour toutes les personnes impliquées dans l'expertise (médecins, juges, avocats, juristes d'assurances et responsables de dossiers) pour des questions spécifiques relatives aux expertises en médecine des assurances.

Hans Zollinger-Kies, Zurich 